Proyecciones Journal of Mathematics

Vol. 33, No 1, pp. 101-122, March 2014.

Universidad Católica del Norte

Antofagasta - Chile

\title{
On the asymptotic behaviour of solutions of certain differential equations of the third order
}

\author{
A. T. Ademola \\ and \\ P. O. Arawomo \\ University of Ibadan, Nigeria \\ Received : February 2013. Accepted : October 2013
}

\begin{abstract}
In this article, Lyapunov second method is used to obtain criteria for uniform ultimate boundedness and asymptotic behaviour of solutions of nonlinear differential equations of the third order. The results obtained in this investigation include and extend some well known results on third order nonlinear differential equations in the literature.
\end{abstract}

Subjclass [2000] : 34D20, 34D40.

Keywords : Third order, Differential equations, Uniform ultimate boundedness, Asymptotic behaviour of solutions, Complete Lyapunov function. 


\section{Introduction}

The investigation of qualitative behaviour of solutions such as stability, convergence, boundedness, oscillation, asymptotic behaviour to mention few, are very important problems in the theory and applications of differential equations. For instance, in applied sciences some practical problems concerning mechanics, engineering technique fields, economy, control theory, physical sciences and so on are associated with second, third, fourth and higher order nonlinear differential equations.

Many interesting results, on the qualitative behaviour of solutions of nonlinear differential equations have been obtained see for examples Reissig, et. al., [21], Rouche et. al., [22] and Yoshizawa [29]. Notable authors that have contributed immensely to the qualitative behaviour of solutions of third order nonlinear differential equations include Ademola et. al., [1, $3,5,6]$ on uniform stability and boundedness of solutions, Afuwape and Adesina [7], Chukwu [9], Ezeilo [10, 11, 13], Ezeilo and Tejumola [14], Swick [24], Tejumola [25] and Tunç [26] on boundedness of solutions, Afuwape and Omeike [19], Ogundare [18] and Tunç [28] on convergence of solutions, Omeike [19], Qian [20], Swick [23] and Tunç [27] on asymptotic behaviour of solutions. Most of these works were done with the aid of Lyapunov functions which are either incomplete or contain signum functions.

However, the problem of boundedness and asymptotic behaviour of solutions of third order differential equations in which the nonlinear (in particular the restoring) terms depend on the independent variable $t$ or multiple of functions of $t$ are scarce. In this work, a complete Lyapunov function was constructed and used, using the direct method of Lyapunov, to study uniform ultimate boundedness and asymptotic behaviour of solutions of the nonlinear non autonomous third order differential equation

$$
\dddot{x}+f(t, x, \dot{x}, \ddot{x}) \ddot{x}+q(t) g(x, \dot{x})+r(t) h(x, \dot{x})=p(t, x, \dot{x}, \ddot{x})
$$

or its equivalent system of first order differential equations

$$
\dot{x}=y, \dot{y}=z, \dot{z}=p(t, x, y, z)-f(t, x, y, z) z-q(t) g(x, y)-r(t) h(x, y),
$$


in which the functions $f, g, h, p, q$ and $r$ are continuous in their respective arguments and the derivatives $f_{t}(t, x, y, z), f_{x}(t, x, y, z), f_{z}(t, x, y, z), g_{x}(x, y)$ $h_{x}(x, y), h_{y}(x, y), q^{\prime}(t)$ and $r^{\prime}(t)$ exist and are continuous for all values of $t, x, y$ and $z$. As usual, condition for uniqueness of solutions of 1.2 will be assumed and $\dot{x}, \ddot{x}, \dddot{x}$ as elsewhere stand for differentiation with respect to $t$. Motivation for this work come from the works of Ademola and Arawomo [2], Omeike [19], Qian [20], Swick [23, 24] and Tunç [27]. The results obtained in this investigation include and generalize the existing results on third order nonlinear differential equations in the literature.

\section{Preliminaries}

Consider the system of the form

$$
\dot{X}=F(t, X)
$$

where $t \in \mathbf{R}^{+}=[0, \infty), X \in \mathbf{R}^{n}, F \in C\left(\mathbf{R}^{+} \times \mathbf{R}^{n}, \mathbf{R}^{n}\right)$ and $\mathbf{R}^{n}$ is the $n$-dimensional Euclidean space.

Definition 2.1. The solutions of 2.1 are uniformly bounded if for any $\alpha_{0}>$ 0 and any $t_{0} \in \mathbf{R}^{+}$, there exists a $\beta\left(\alpha_{0}\right)>0$ such that $\left\|X_{0}\right\|<\alpha_{0}$ implies that

$$
\left\|X\left(t, t_{0}, X_{0}\right)\right\|<\beta\left(\alpha_{0}\right) .
$$

Definition 2.2. The solutions of 2.1 are uniformly ultimately bounded for bound $\beta$, if there exists a $\beta>0$ and if corresponding to any $\alpha_{0}>0$ there exists a $T\left(\alpha_{0}\right)>0$ such that $\left\|X_{0}\right\|<\alpha_{0}$ implies that

$$
\left\|X\left(t, t_{0}, X_{0}\right)\right\|<\beta
$$

for all $t \geq t_{0}+T\left(\alpha_{0}\right)$.

Definition 2.3. (i) A function $\phi: \mathbf{R}^{+} \rightarrow \mathbf{R}^{+}$, continuous, strictly increasing with $\phi(0)=0$ is said to be a function of class $\mathbf{K}$ for such function, we shall write $\phi \in \mathbf{K}$.

(ii) If in addition to (i) $\phi(r) \rightarrow+\infty$ as $r \rightarrow \infty$, $\phi$ is said to be a function of class $\mathbf{K}^{*}$.

Definition 2.4. A function $W(X)$ defined for $X \in Q(Q$ an open set in $\left.\mathbf{R}^{n}\right)$ is said to be positive definite with respect to a set $\Omega$ if $W(X)=0$ for 
all $X \in \Omega$ and if corresponding to each $\epsilon>0$ and each compact set $Q^{*}$ in $Q$ there exist a positive number $\delta\left(\epsilon, Q^{*}\right)$ such that

$$
W(X) \geq \delta\left(\epsilon, Q^{*}\right)
$$

for all $X \in Q^{*} \backslash N(\epsilon, \Omega)$.

Next, we give some results which play significant role in the proofs of our results.

Lemma 2.5. [16] Suppose that $V(t, X)$ is a scalar function with continuous first partial derivatives which satisfies the following conditions:

(i) $V(t, X) \geq a(\|X\|)$, for all $(t, X) \in \mathbf{R}^{+} \times \mathbf{R}^{n}, a \in \mathbf{K}^{*}$

(ii) $\dot{V}_{2.1}(t, X) \leq-\epsilon<0$ for all $(t, X) \in \mathbf{R}^{+} \times \mathbf{R}^{n}$.

Then the solutions of 2.1 are ultimately bounded.

Lemma 2.6. [29] Suppose that there exists a Lyapunov function $V(t, X)$ defined on $\mathbf{R}^{+},\|X\| \geq \rho$ were $\rho>0$ may be large which satisfies the following conditions:

(i) $a(\|X\|) \leq V(t, X) \leq b(\|X\|), a \in \mathbf{K}^{*}$ and $b \in \mathbf{K}$;

(ii) $\dot{V}_{2.1} \leq 0$ for all $(t, X) \in \mathbf{R}^{+} \times \mathbf{R}^{n}$.

Then the solutions of 2.1 are uniformly bounded.

Lemma 2.7. [29] If in addition to hypothesis (i) of Lemma 2.6, $\dot{V}_{2.1} \leq$ $-c(\|X\|), c \in \mathbf{K}$ for all $(t, X) \in \mathbf{R}^{+} \times \mathbf{R}^{n}$.

Then the solutions of 2.1 are uniformly ultimately bounded.

Next, consider the system of equation

$$
\dot{X}=F(t, X)+G(t, X)
$$

where $F(t, X)$ and $G(t, X)$ are continuous on $\mathbf{R}^{+} \times Q, Q$ an open set in $\mathbf{R}^{n}$. We have the following result. 
Lemma 2.8. [29] Suppose that there exist a Lyapunov function $V(t, X)$ defined on $\mathbf{R}^{+} \times \mathbf{R}^{n}$ such that

$$
\dot{V}_{2.2}(t, X) \leq-W(X)
$$

where $W(X)$ is positive definite with respect to a closed set $\Omega$ in the space $\mathbf{R}^{n}$.

Moreover suppose that $F(t, X)$ satisfies conditions:

(i) $F(t, X)$ tends to a function $H(X)$ for $X \in \Omega$ as $t \rightarrow \infty$ and on any compact set in $\Omega$ this convergence is uniform;

(ii) Corresponding to each $\epsilon>0$ and each $Y \in \Omega$ there exists a $\delta(\epsilon, Y)>0$ and a $T(\epsilon, Y)>0$ such that if $\|X-Y\|<\delta(\epsilon, Y)$ and $t \geq T(\epsilon, Y)$, we have

$$
\|F(t, X)-F(t, Y)\|<\epsilon .
$$

Then every bounded solution of 2.1 approaches the largest semi-invariant set of the system $\dot{X}=H(X), X \in \Omega$ as $t \rightarrow \infty$.

\section{Statement of Results}

We define the Lyapunov function $V \equiv V(t, x(t), y(t), z(t))$ as

$$
V=e^{-P_{*}(t)} U \text {, }
$$

where

$$
P_{*}(t)=\int_{0}^{t}|p(\mu, x, y, z)| d \mu
$$

and $U \equiv U(t, x(t), y(t), z(t))$ is the function defined by

$$
\begin{aligned}
& 2 U=2(\alpha+a) r(t) \int_{0}^{x} h(\xi, 0) d \xi+4 q(t) \int_{0}^{y} g(x, \tau) d \tau+4 r(t) y h(x, 0) \\
& +2(\alpha+a) y z+2 z^{2}+2(\alpha+a) \int_{0}^{y} \tau f(t, x, \tau, 0) d \tau+\beta y^{2}+b \beta x^{2} \\
& +2 \mathrm{a} \beta x y+2 \beta x z(3.3)
\end{aligned}
$$

where $\alpha$ and $\beta$ are positive fixed constants satisfying

$$
b^{-1} c<\alpha<a
$$


and

$$
0<\beta<\min \left\{(a b-c) a^{-1}, \delta_{1}(a b-c) \eta_{0}^{-1}, \frac{1}{2}(a-\alpha) \eta_{1}^{-1}\right\}
$$

where $\eta_{0}:=1+a+\delta_{0}^{-1} \delta_{1}^{-1}\left(q(t) \frac{g(x, y)}{y}-b\right)^{2}$ and $\eta_{1}:=1+\delta_{0}^{-1} \delta_{1}^{-1}(f(t, x, y, z)-$ $a)^{2}$.

We have the following results.

Theorem 3.1. Further to the basic assumptions on the functions $f, g, h, p, q$ and $r$ defined in 1.2, suppose that $a, a_{1}, b, b_{1}, c, \delta_{0}, \delta_{1}$ are positive constants and for all $t \geq 0$ :

(i) $a \leq f(t, x, y, z) \leq a_{1}$ for all $x, y, z$;

(ii) $b \leq \frac{g(x, y)}{y} \leq b_{1}$ for all $x$ and $y \neq 0$;

(iii) $h(0,0)=0, \delta_{0} \leq \frac{h(x, y)}{x}$ for all $x \neq 0$ and $y$;

(iv) $\delta_{1} \leq r(t) \leq q(t), \dot{q}(t) \leq \dot{r}(t) \leq 0$;

(v) $f_{t}(t, x, y, 0) \leq 0, y f_{x}(t, x, y, 0) \leq 0, y f_{z}(t, x, y, z) \geq 0, g_{x}(x, y) \leq 0$, $y z h_{y}(x, y) \geq 0, h_{x}(x, 0) \leq c$ for all $x, y, z$ and $a b>c ;$

(vi) $\int_{0}^{\infty}|p(t, x, y, z)| d t<\infty$.

Then the solutions of 1.2 are uniformly ultimately bounded.

Theorem 3.2. Under the assumption of Theorem 3.1, the solutions of 1.2 are ultimately bounded and satisfies

$$
|x(t)| \leq D_{0}, \quad|y(t)| \leq D_{0}, \quad|z(t)| \leq D_{0} \forall t \geq 0
$$

where $D_{0}=D_{0}\left(a, b, c, \delta_{0}, \alpha, \beta\right)>0$ is a constant.

If $p(t, x, y, z) \equiv 0$, Eq. 1.2 becomes

$$
\dot{x}=y, \dot{y}=z, \dot{z}=-f(t, x, y, z) z-q(t) g(x, y)-r(t) h(x, y),
$$

with the following results

Corollary 3.3. If $g(0,0)=0$ and hypotheses (i)-(v) of Theorem 3.1 hold, then 
(i) the trivial solution of 3.7 is uniformly asymptotically stable.

(ii) the solution $(x(t), y(t), z(t))$ of 3.7 is uniformly bounded and satisfies

$$
x(t) \rightarrow 0, \quad y(t) \rightarrow 0, \quad z(t) \rightarrow 0 \text { as } t \rightarrow \infty .
$$

Furthermore, if $p(t, x, y, z) \equiv p(t) \neq 0, p: \mathbf{R}^{+} \rightarrow \mathbf{R}$ Eq. 1.2 reduces to

$$
\dot{x}=y, \dot{y}=z, \dot{z}=p(t)-f(t, x, y, z) z-q(t) g(x, y)-r(t) h(x, y),
$$

with the following result.

Corollary 3.4. If hypotheses (i)-(v) of Theorem 3.1 hold and in addition $\int_{0}^{\infty}|p(t)| d t<\infty$, then the solutions of 3.8 are uniformly ultimately bounded.

Remark 3.5. (i) Whenever: $f(t, x, y, z) \equiv a, g(x, y) \equiv b y, a>0$ and $b>0$ are constants, $h(x, y) \equiv h(x), p(t, x, y, z) \equiv p(t)$, and $q(t) \equiv$ $1 \equiv r(t)$ system 1.2 reduces to that studied by Ezeilo [11, 13] and Tejumola [25]. Thus our results include and generalize theirs.

(ii) When $f(t, x, y, z) \equiv a a>0$ is a constant, $g(x, y) \equiv y g(x)$ or $\phi_{2}(y)$, $h(x, y) \equiv h(x)$ or $\phi_{3}(x), p(t, x, y, z) \equiv p(t)$ and $q(t) \equiv 1 \equiv r(t)$ system 1.2 specializes to that discussed by Ezeilo [12], Ezeilo and Tejumola [14] and Swick [23, 24].

(iii) In the case when $f(t, x, y, z) \equiv f(t, x, y)$ and $g(x, y) \equiv g(y)$ system 1.2 reduces to that discussed by Ademola and Arawomo [2], Swick [24] and Ezeilo [15]. Furthermore, hypotheses and conclusion of Theorem 3.1 coincide with that in [2] Theorem 3. Hence our results include and improve [2, 15] and [24].

(iv) The situation when $f(t, x, y, z) \equiv f(z), g(x, y) \equiv g(y), h(x, y) \equiv h(x)$ and $q(t) \equiv 1 \equiv r(t)$ some of our assumptions coincide with that discussed by Ademola et. al., [5, 6], the hypothesis that $H(x) \rightarrow+\infty$ as $|x| \rightarrow \infty$ in $[6,15]$ and $-L \leq \dot{q}(t) \leq \dot{r}(t)$ in [24] are not required here.

(v) When $g(x, \dot{x})=g(\dot{x})$ and $h(x, \dot{x})=h(x)$ system 1.2 reduces to that considered by Ademola and Arawomo [4]. The position of the functions $\dot{q}(t)$ and $\dot{r}(t)$ is swaped here. 
In what follows, we shall state and proof a result that would be useful in the proofs of the main and the subsequent results.

Lemma 3.6. Under the assumptions of Theorem 3.1, there exist positive constants $D_{0}=D_{0}\left(a, b, c, \alpha, \beta, \delta_{0}, \delta_{1}, P_{0}\right)$ and

$D_{1}=D_{1}\left(a, b, c, a_{1}, b_{1}, \alpha, \beta, q_{0}, r_{0}\right)$ such that for the function $V$ defined in 3.3, we have

$$
D_{0}\left(x^{2}(t)+y^{2}(t)+z^{2}(t)\right) \leq V(t, x, y, z) \leq D_{1}\left(x^{2}(t)+y^{2}(t)+z^{2}(t)\right)
$$

and that

$$
V(t, x, y, z) \rightarrow+\infty \text { as } x^{2}(t)+y^{2}(t)+z^{2}(t) \rightarrow \infty .
$$

Furthermore, there exists a constant $D_{2}=D_{2}\left(a, b, c, \alpha, \beta, \delta_{0}, \delta_{1}\right)$ such that along a solution $(x(t), y(t), z(t))$ of 1.2 ,

$$
\dot{V} \equiv \frac{d}{d t} V(t, x, y, z) \leq-D_{2}\left(x^{2}(t)+y^{2}(t)+z^{2}(t)\right)
$$

for all $t \geq 0, x, y$ and $z$.

Proof of Lemma 3.6. Since $h(0,0)=0$, the function $U$ defined in 3.1 can be rearranged in the form

$$
\begin{gathered}
U=\frac{r(t)}{b} \int_{0}^{x}\left[(\alpha+a) b-2 h_{x}(\xi, 0)\right] h(\xi, 0) d \xi+\frac{1}{2}(\alpha y+z)^{2}+\frac{\beta}{2} y^{2} \\
+2 \mathrm{r}(\mathrm{t}) \int_{0}^{y}\left[\frac{q(t)}{r(t)} \frac{g(x, \tau)}{\tau}-b\right] \tau d \tau+\frac{r(t)}{b}(b y+h(x, 0))^{2}+\frac{\beta}{2}(b-\beta) x^{2} \\
+\int_{0}^{y}\left[(\alpha+a) f(t, x, \tau, 0)-\left(\alpha^{2}+a^{2}\right)\right] \tau d \tau+\frac{1}{2}(\beta x+a y+z)^{2} .(3.12)
\end{gathered}
$$


By Theorem 3.1, we have $h(x, y) \geq \delta_{0} x, h_{x}(x, 0) \leq c, g(x, y) \geq b y$, $f(t, x, y, 0) \geq a, r(t) \geq \delta_{1}$ and $q(t) \geq r(t)$. These estimates and 3.12 yields

$$
\begin{gathered}
U \geq \frac{1}{2}\left[(\alpha b-c+a b-c) b^{-1} \delta_{0} \delta_{1}+\beta(b-\beta)\right] x^{2}+\frac{1}{2}[\alpha(a-\alpha)+\beta] y^{2} \\
+\frac{\delta_{1}}{b}\left(\delta_{0} x+b y\right)^{2}+\frac{1}{2}(\beta x+a y+z)^{2}+\frac{1}{2}(\alpha y+z)^{2} .
\end{gathered}
$$

From estimates 3.4 and 3.5, the quadratic in the right hand side of this inequality is positive definite. Hence there exists a positive constant $K \equiv K\left(a, b, c, \alpha, \beta, \delta_{0}, \delta_{1}\right)$ such that

$$
U \geq K\left(x^{2}+y^{2}+z^{2}\right)
$$

for all $t \geq 0, x, y$ and $z$. In view of hypothesis (vi) of Theorem 3.1, there exists a positive constant $P_{0}<\infty$ such that

$$
P_{*}(t) \leq P_{0} \text { for all } t \geq 0 \text {. }
$$

Now from estimates 3.13 and 3.14, Eq. 3.1 becomes

$$
V \geq K_{0}\left(x^{2}+y^{2}+z^{2}\right)
$$

for all $t \geq 0, x, y$ and $z$, where $K_{0} \equiv K e^{-P_{0}}>0$. Hence, the lower inequality in 3.9 is established. Also, from 3.15 we have

$$
V(t, x, y, z) \rightarrow+\infty \text { as } x^{2}+y^{2}+z^{2} \rightarrow \infty .
$$

Moreover, from the hypotheses of Theorem 3.1, 3.1 yields

$$
\begin{aligned}
V \leq & \frac{1}{2}\left[(\alpha+a) c r_{0}+b \beta\right] x^{2}+\frac{1}{2}\left[(\alpha+a) a_{1}+\beta+2 b_{1} q_{0}\right] y^{2} \\
& +z^{2}+\left(a \beta+2 c r_{0}\right)|x y|+\beta|x z|+(\alpha+a)|y z| .
\end{aligned}
$$

Applying the inequalities $2|x y| \leq x^{2}+y^{2}, 2|x z| \leq x^{2}+z^{2}$ and $2|y z| \leq$ $y^{2}+z^{2}$, it follows that

$$
V \leq K_{1}\left(x^{2}+y^{2}+z^{2}\right)
$$

for all $t \geq 0, x, y$ and $z$, where $K_{1}:=\frac{1}{2} \max \left\{K_{11}, K_{12}, K_{13}\right\}, K_{11}:=$ $(2+\alpha+a) c r_{0}+(1+a+b) \beta, K_{12}:=(\alpha+a)\left(1+a_{1}\right)+(1+a) \beta+2\left(b_{1} q_{1}+c r_{0}\right)$ 
and $K_{13}:=\alpha+\beta+a+2$.

Next, let $(x(t), y(t), z(t))$ be any solution of 1.2 , the derivative of the function $V$ with respect to $t$ along a solution of 1.2 is

$$
\dot{V}_{1.2}=-e^{-P_{*}(t)}\left[U|p(t, x, y, z)|-\dot{U}_{1.2}\right]
$$

where $P_{*}(t)$ and $U$ are defined in 3.2 and 3.3 respectively and on simplifying

$$
\begin{array}{r}
\dot{U}_{1.2}=a \beta y^{2}+2 \beta y z+(\beta x+(\alpha+a) y+2 z) p(t, x, y, z) \\
+\mathrm{W}_{1}+W_{2}-W_{3}-W_{4} x^{2}-W_{5} y^{2}-W_{6} z^{2} \\
-\beta\left(q(t) \frac{g(x, y)}{y}-b\right) x y-\beta(f(t, x, y, z)-a) x z(3.19)
\end{array}
$$

where:

$$
\begin{aligned}
& \mathrm{W}_{1}:=(\alpha+a) \dot{r}(t) \int_{0}^{x} h(\xi, 0) d \xi+2 \dot{q}(t) \int_{0}^{y} g(x, \tau) d \tau+2 \dot{r}(t) y h(x, 0) ; \\
& \mathrm{W}_{2}:=(\alpha+a) \int_{0}^{y} \tau f_{t}(x, \tau, 0) d \tau+2 q(t) y \int_{0}^{y} g_{x}(x, \tau) d \tau \\
& +(\alpha+a) y \int_{0}^{y} \tau f_{x}(t, x, \tau, 0) d \tau \\
& \mathrm{W}_{3}:=r(t)[2 z+(\alpha+a) y][h(x, y)-h(x, 0)] \\
& +(\alpha+a)[f(t, x, y, z)-f(t, x, y, 0)] \\
& \mathrm{W}_{4}:=\beta r(t) \frac{h(x, y)}{x} ; W_{5}:=r(t)\left[(\alpha+a) \frac{q(t)}{r(t)} \frac{g(x, y)}{y}-2 h_{x}(x, 0)\right] \text { and } \\
& \mathrm{W}_{6}:=2 f(t, x, y, z)-(\alpha+a) .
\end{aligned}
$$

Applying the hypotheses of Theorem 3.1, we have the following estimates for

$W_{i},(i=1, \cdots, 6)$ :

$$
W_{1} \leq 0, \quad W_{2} \leq 0 \text { forall } t \geq 0, x \text { and } y
$$

Also,

$$
W_{3} \geq \delta_{1}[2 z+(\alpha+a) y] y h_{y}\left(x, \theta_{1} y\right)+(\alpha+a) y z^{2} f_{z}\left(t, x, y, \theta_{2} z\right) \geq 0
$$


where $0 \leq \theta_{i} \leq 1(i=1,2)$, but $W_{3}=0$ when $y=0=z$.

$$
W_{4} \geq \beta \delta_{0} \delta_{1}, \quad W_{5} \geq \delta_{1}((\alpha+a) b-2 c) \text { and } W_{6} \geq a-\alpha
$$

for all $t \geq 0, x, y$ and $z$. Using estimates $W_{i},(i=1, \cdots, 6)$ in 3.19 noting that $x \leq|x|$, we have

$$
\begin{gathered}
\dot{U}_{1.2} \leq-\frac{1}{2} \beta \delta_{0} \delta_{1} x^{2}-\delta_{1}\left[\alpha b-c+a b-c-\delta_{1}^{-1} \beta(1+a)\right] y^{2} \\
-(\mathrm{a}-\alpha-\beta) z^{2}-W_{7}-W_{8}+(\beta|x|+(\alpha+a)|y|+2|z|)|p(t, x, y, z)|
\end{gathered}
$$

where $W_{7}:=\frac{1}{4} \beta \delta_{0} \delta_{1} x^{2}+\beta\left[q(t) \frac{g(x, y)}{y}-b\right] x y$ and $W_{8}:=\frac{1}{4} \beta \delta_{0} \delta_{1} x^{2}+$ $\beta[f(t, x, y, z)-a] x z$.

Completing the squares in $W_{7}$ and $W_{8}$, estimate 3.20 yields

$\dot{U}_{1.2} \leq-\frac{1}{2} \beta \delta_{0} \delta_{1} x^{2}-\delta_{1}(\alpha b-c) y^{2}-\frac{1}{2}(a-\alpha) z^{2}$

$-\left\{\delta_{1}(a b-c)-\beta\left[1+a+\delta_{0}^{-1} \delta_{1}^{-1}\left(q(t) \frac{g(x, y)}{y}-b\right)^{2}\right]\right\} y^{2}$
$-\left\{\frac{1}{2}(a-\alpha)-\beta\left[1+\delta_{0}^{-1} \delta_{1}^{-1}(f(t, x, y, z)-a)^{2}\right]\right\} z^{2}$
$+(\beta|x|+(\alpha+a)|y|+2|z|)|p(t, x, y, z)|$.

Using estimates 3.4 and 3.5, with the fact that $(|x|+|y|+|z|)^{2} \leq$ $3\left(x^{2}+y^{2}+z^{2}\right)$, we obtain

$$
\dot{U}_{1.2} \leq-K_{2}\left(x^{2}+y^{2}+z^{2}\right)+K_{3}\left(x^{2}+y^{2}+z^{2}\right)^{1 / 2}|p(t, x, y, z)|,
$$

for all $t \geq 0, x, y$ and $z, K_{2}:=\min \left\{\frac{1}{2} \beta \delta_{0} \delta_{1}, \delta_{1}(\alpha b-c), \frac{1}{2}(a-\alpha)\right\}>0$ and $K_{3}:=3^{1 / 2} \max \{\beta, \alpha+a, 2\}$. Using estimates 3.13 and 3.21 in 3.18 choosing $\left(x^{2}+y^{2}+z^{2}\right)^{1 / 2} \geq K^{-1} K_{3}$ and by condition (vi) of Theorem 3.1, we see that

$$
\dot{V}_{1.2} \leq-K_{4}\left(x^{2}+y^{2}+z^{2}\right)
$$

for all $t \geq 0, x, y$ and $z$, where $K_{4} \equiv K_{2} e^{-P_{*}(\infty)}$. This completes the proof of the Lemma. 
Proof of Theorem 3.1. The proof of this theorem depends on the function $V$ defined in 3.1. From estimates 3.15, 3.16, 3.17 and 3.22 hypotheses of Lemma 2.7 hold. Hence, by Lemma 2.7 the solutions of 1.2 are uniformly ultimately bounded. This completes the proof of the theorem.

Proof of Theorem 3.2. The proof of this theorem depends on the function $V$ defined in 3.1. From estimates 3.15 and 3.16, hypothesis (i) of Lemma 2.5 holds. Also, by 3.22 , we have

$$
\dot{V}_{1.2} \leq-K_{5}<0
$$

provided that $x^{2}+y^{2}+z^{2} \geq K_{4}^{-1} K_{5}$, so that by 3.23 assumption (ii) of Lemma 2.5 follows, thus the solutions of 1.2 are ultimately bounded. For the proof of 3.6, see [9], hence it is omitted this completes the proof of the theorem.

Theorem 3.7. If the hypotheses of Theorem 3.1 hold true, then the solution $(x(t), y(t), z(t))$ of 1.2 is uniformly bounded and satisfies

$$
\lim _{t \rightarrow \infty} x(t)=0, \quad \lim _{t \rightarrow \infty} y(t)=0, \quad \lim _{t \rightarrow \infty} z(t)=0 .
$$

Corollary 3.8. If the hypotheses of Corollary 3.4 are satisfied, then the solutions of 3.8 are uniformly bounded and satisfy 3.24 .

Remark 3.9. (i) If $f(t, x, y, z) \equiv a a>0$ is a constant or $p(t)$ or $f(t, x, y), g(x, y) \equiv g(y), p(t, x, y, z) \equiv 0$ or $e(t)$ and $r(t) \equiv 1$, system 1.2 reduces to that studied by Swick in [23] and [24]. Restriction imposed on $e(t)$ in [23] is relaxed in ours.

(ii) Whenever $f(t, x, y, z) \equiv a a>0$ is a constant or $e(t), g(x, y) \equiv y g(x)$, $q(t) \equiv 1$ and $r(t) \equiv 1$ system 1.2 specializes to that discussed by Nakashima [17]. Hence our result generalizes his.

Proof of Theorem 3.7 Under the hypotheses of Theorem 3.7 estimates $3.15,3.16,3.17$ and 3.22 hold for the function $V$ defined in 3.1, thus by Lemma 2.6, solutions of 1.2 are uniformly bounded. Next, from estimate 3.22 setting $W(X) \equiv K_{4}\left(x^{2}+y^{2}+z^{2}\right)$, clearly, $W(X) \geq 0$ for all $X \in \mathbf{R}^{3}$. Consider the set

$$
\Omega:=\left\{X=(x, y, z) \in \mathbf{R}^{3} \mid W(X)=0 .\right\}
$$


Since $W(X)$ is continuous, set $\Omega$ is closed and $W(X)$ is positive definite with respect to $\Omega$ and

$$
\dot{V}_{1.2} \leq-W(X) \forall(t, X) \in \mathbf{R}^{+} \times \mathbf{R}^{3}
$$

so that estimate 2.3 holds. Moreover, system 1.2 can be written in the form

$$
\dot{X}=F(t, X)+G(t, X)
$$

where $X=(x, y, z)^{T}, F(t, X)=(y, z,-f(t, x, y, z) z-q(t) g(x, y)-r(t) h(x, y))^{T}$ and $G(t, X)=(0,0, p(t, x, y, z))^{T}$. From the continuity and boundedness of the functions $f, g, h, q$ and $r, F(t, X)$ is bounded for all $(t, X) \in \mathbf{R}^{+} \times \mathbf{R}^{3}$. Next, from the hypotheses of Theorem 3.7 $F(t, X) \rightarrow H(X)$ as $t \rightarrow \infty$ and since $W(X)=0$ on $\Omega$, it follows, from 1.2 and the fact that $h(0,0)=0=$ $g(0,0)$, that the largest semi invariant set of $\dot{X}=H(X), X \in \Omega$ as $t \rightarrow \infty$ is the origin. Hence estimates 3.24 follows immediately. This completes the proof of the theorem.

Proof of Corollary 3.8 The proof of this corollary is similar to the proof of Theorem 3.7, hence it is omitted.

\section{Some Special Cases}

In this section, we discuss uniform ultimate boundedness and asymptotic behaviour of solutions of some special cases of 1.2. When $h(x, y) \equiv h(x)$, 1.2 reduces to

$$
\dot{x}=y, \dot{y}=z, \dot{z}=p(t, x, y, z)-f(t, x, y, z) z-q(t) g(x, y)-r(t) h(x),
$$

where $h: \mathbf{R} \rightarrow \mathbf{R}, h^{\prime}(x)$ exists and is continuous for all $x$. We obtain the following results.

Theorem 4.1. If assumptions (i), (ii), (iv) and (vi) of Theorem 3.1 hold and in addition suppose that $\delta>0$ and $c_{0}>0$ are constants and for all $t \geq 0$ : 
(i) $h(0)=0, \delta \leq \frac{h(x)}{x}$ for all $x \neq 0$

(ii) $g_{x}(x, y) \leq 0, f_{t}(t, x, y, 0) \leq 0, y f_{x}(t, x, y, 0) \leq 0, y f_{z}(t, x, y, z) \geq 0$, $h^{\prime}(x) \leq c_{0}$ for all $x, y$ and $z$.

Then the solutions of 4.1 are uniformly ultimately bounded.

Proof. Using 3.1, with $h(x, y) \equiv h(x), \delta_{0} \equiv \delta$ and $c \equiv c_{0}$ estimates 3.15, $3.16,3.17$ and 3.22 hold. The conclusion of the remaining part of the proof follows the steps in the proof of Theorem 3.1. Hence, it is omitted.

Theorem 4.2. If assumptions: (i), (ii), (iv) of Theorem 3.1; (i)', (ii)' of Theorem 4.1 are satisfied and in addition

$$
|p(t, x, y, z)| \leq p_{1}(t)+p_{2}(t)(|x|+|y|+|z|)
$$

where $p_{1}(t)$ and $p_{2}(t)$ are nonnegative continuous functions satisfying $p_{1}(t) \leq$ $P_{1}, 0 \leq P_{1}<\infty$ and there exists $\epsilon>0$ such that $0 \leq p_{2}(t) \leq \epsilon$. Then the solutions of 4.1 are uniformly ultimately bounded.

Proof. The proof of this result depends on the function $U$ defined in 3.3, 3.4 and 3.5. Setting $h(x, y) \equiv h(x), \delta_{0} \equiv \delta$ and $c \equiv c_{0}$ estimates 3.13 and 3.16 are satisfied for the function $U$. Also, if $t=0$ in 3.2, the function $V$ coincides with $U$ and hence, estimate 3.17 hold true for the function $U$. Furthermore, from 3.21 and 4.2 , we have

$$
\dot{U}_{1.2} \leq-\left(K_{6}-3^{1 / 2} K_{3} \epsilon\right)\left(x^{2}+y^{2}+z^{2}\right)+K_{3} P_{1}\left(x^{2}+y^{2}+z^{2}\right)^{1 / 2}
$$

where $K_{6}:=\min \left\{\frac{1}{2} \beta \delta \delta_{1}, \delta\left(a b-c_{0}\right), \frac{1}{2}(a-\alpha)\right\}>0$. Choosing $\epsilon<3^{-1 / 2} K_{3}^{-1} K_{6}$ and $\left(x^{2}+y^{2}+z^{2}\right)^{1 / 2} \geq 2 K_{3} K_{7}^{-1} P_{1}$, we see that

$$
\dot{U}_{1.2} \leq-K_{8}\left(x^{2}+y^{2}+z^{2}\right)
$$

for all $t \geq 0, x, y$ and $z$, where $K_{7}:=K_{6}-3^{1 / 2} K_{3} \epsilon>0$ and $K_{8}:=\frac{1}{2} K_{7}>0$ are constants. From 3.13, 3.16, 3.17 and 4.3, the hypotheses of Lemma 2.7 hold, this completes the proof of the theorem. 
Corollary 4.3. Under the hypotheses of Theorem 4.1 and Theorem 4.2, the solutions of 4.1 are ultimately bounded and satisfy 3.6 for all $t \geq 0$.

Remark 4.4. (i) When $f(t, x, y, z) \equiv \psi(x, y), p(t, x, y, z) \equiv p(t), q(t) \equiv$ 1 and $r(t) h(x)=0$ our results include and extends the situation given by Omeike [19] Theorem 1 and Qian [20] Theorem 1.1

(ii) In the case when $f(t, x, y, z) \equiv f(x, y), q(t) \equiv 1$ and $r(t) \equiv 1$ system 4.1 reduces to that discussed by Chukwu [9].

Next, if $p(t, x, y, z) \equiv p(t) \neq 0,4.1$ specializes to

$$
\dot{x}=y, \dot{y}=z, \dot{z}=p(t)-f(t, x, y, z) z-q(t) g(x, y)-r(t) h(x),
$$

where $p: \mathbf{R}^{+} \rightarrow \mathbf{R}$, we obtain the following result.

Theorem 4.5. Assuming assumptions: (i), (ii), (iv) of Theorem 3.1; (i)', (ii) $^{\prime}$ of Theorem 4.1 and in addition $\int_{0}^{\infty}|p(t)| d t<\infty$. Then the solution $x(t)$ of 4.4, its first and second derivatives are ultimately uniformly bounded.

Proof. Similar to the proof of Theorem 3.1, hence it is omitted.

Theorem 4.6. In addition to the hypotheses of Theorem 4.1, suppose that $g(0,0)=0$, then the solutions $x(t)$ of 4.1, its first and second derivatives are uniformly bounded and satisfy 3.24 .

Proof. Let $(x(t), y(t), z(t))$ be any solution of 4.1. Employing the function $V$ used in the proof of Theorem 4.1, the proof of the theorem can be established in a manner similar to the proof of Theorem 3.7, hence it is omitted.

Theorem 4.7. Suppose that $g(0,0)=0$ and the assumptions of Theorem 4.5 hold, then the solution $x(t)$ of 4.4, its first and second derivatives are uniformly bounded and satisfy 3.15 .

Proof. Employing the function $V$ defined in 3.1 with $p(t, x, y, z) \equiv p(t)$ and $h(x, y) \equiv h(x)$. Using an argument similar to that in the proof of Theorem 3.7, the solutions of 4.4 are uniformly bounded and satisfy 3.15. 
Example 4.8. As a special case of equation 1.1, consider the following third order nonlinear ordinary differential equation

$$
\begin{gathered}
\dddot{x}+4 \ddot{x}+\frac{\ddot{x}}{1+t^{2}+|x \dot{x}|+\exp (1 /(1+|\dot{x} \ddot{x}|))} \\
+\left(\frac{1}{2}+\frac{1}{1+t^{2}}\right)\left(3 \dot{x}+\frac{\dot{x}}{1+|x \dot{x}|}\right) \\
+\left(\frac{1}{2}+\frac{1}{2+t^{2}}\right)\left(5 x+\frac{x}{1+\exp \left(1 /\left(1+|x| \dot{x}^{2}\right)\right)}\right) \\
=\frac{1}{1+t^{2}+x^{2}+\dot{x}^{2}+\ddot{x}^{2}}
\end{gathered}
$$

4.5 is equivalent to

$$
\begin{gathered}
\dot{x}=y, \quad \dot{y}=z, \quad \dot{z}=\frac{1}{1+t^{2}+x^{2}+y^{2}+z^{2}}-4 z \\
-\frac{z}{1+t^{2}+|x y|+\exp (1 /(1+|y z|))}-\left(\frac{1}{2}+\frac{1}{1+t^{2}}\right)\left(3 y+\frac{y}{1+|x y|}\right) \\
-\left(\frac{1}{2}+\frac{1}{2+t^{2}}\right)\left(5 x+\frac{x}{1+\exp \left(1 /\left(1+|x| y^{2}\right)\right)}\right)
\end{gathered}
$$

Comparing 1.2 and 4.6, we have the following:

(a) the function $f(t, x, y, z)$ is defined as

$$
4-\frac{1}{1+t^{2}+|x y|+\exp (1 /(1+|y z|))}
$$

(i) Now, since $0 \leq \frac{1}{1+t^{2}+|x y|+\exp (1 /(1+|y z|))} \leq 1$ for all $t \geq 0, x, y$ and $z$, it follows that

$$
4 \leq f(t, x, y, z) \leq 5
$$

for all $t \geq 0, x, y$ and $z$, where $a=4>0$ and $a_{1}=5>0$. 
(ii) From 4.7, we have

$$
f_{t}(t, x, y, z)=\frac{-2 t}{\left[1+t^{2}+|x y|+\exp (1 /(1+|y z|))\right]^{2}} \leq 0
$$

for all $t \geq 0, x, y$ and $z$.

(iii) The derivative of the function in 4.7 with respect to $x>0$ is

$$
f_{x}(t, x, y, z)=\frac{-|y|}{\left[1+t^{2}+|x y|+\exp (1 /(1+|y z|))\right]^{2}}
$$

and

$$
y f_{x}(t, x, y, z)=\frac{-y^{2}}{\left[1+t^{2}+|x y|+\exp (1 /(1+|y z|))\right]^{2}} \leq 0
$$

for all $t \geq 0, x, y$ and $z$.

(iv) Also, if $z>0$

$$
y f_{z}(t, x, y, z)=\frac{y^{2}}{\left[1+t^{2}+|x y|+\exp (1 /(1+|y z|))\right]^{2}} \geq 0
$$

for all $t \geq 0, x, y$ and $z$.

(b) the function $g(x, y)$ is

$$
3 y+\frac{y}{1+|x y|}
$$

(i) clearly $g(0,0)=0$,

(ii) since $0 \leq \frac{1}{1+|x y|} \leq 1$ for all $x$ and $y$, it follows that

$$
3 \leq \frac{g(x, y)}{y} \leq 4
$$

for all $x$ and $y \neq 0$, where $b=3>0$ and $b_{1}=4>0$.

(iii) For $x>0$, we have

$$
g_{x}(x, y)=\frac{-y^{2}}{\left[1+\left.|x y|\right|^{2}\right.} \leq 0
$$

for all $x$ and $y$. 
(c) the function $h(x, y)$ is defined as

$$
5 x+\frac{x}{1+\exp \left(1 /\left(1+|x| y^{2}\right)\right)}
$$

from which we have the following estimates:

(i) clearly, $h(0,0)=0$,

(ii) since $0 \leq \frac{1}{1+\exp \left(1 /\left(1+|x| y^{2}\right)\right)}$ for all $x$ and $y$ it follows that

$$
\frac{h(x, y)}{x} \geq 5
$$

for all $x \neq 0$ and $y$, where $\delta_{0}=5>0$.

(iii) Furthermore,

$$
h_{x}(x, y)-5=\frac{\left[1+|x| y^{2}\right]^{2}\left[1+e^{u}\right]+|x| y^{2} e^{u}}{\left[1+|x| y^{2}\right]^{2}\left[1+e^{u}\right]^{2}}
$$

where $u=\frac{1}{1+|x| y^{2}}$. Since

$$
\frac{\left[1+|x| y^{2}\right]^{2}\left[1+e^{u}\right]+|x| y^{2} e^{u}}{\left[1+|x| y^{2}\right]^{2}\left[1+e^{u}\right]^{2}} \leq 1
$$

for all $x$ when $y=0$, it follows that

$$
h_{x}(x, 0) \leq 6
$$

for all $x$ where $c=5>0$ and $a b>c$ implies that $2>1$.

(iv) Also,

and

$$
h_{y}(x, y)=\frac{2 x^{2} y e^{u}}{\left[1+e^{u}\right]^{2}}
$$

$$
y z h_{y}(x, y)=\frac{2 x^{2} y^{2} z e^{u}}{\left[1+e^{u}\right]^{2}} \geq 0
$$

for all $x, y$ and $z>0$,

(d) the functions $q(t)$ and $r(t)$ are

$$
\frac{1}{2}+\frac{1}{1+t^{2}} \text { and } \frac{1}{2}+\frac{1}{2+t^{2}}
$$

respectively. 
(i) Since $\frac{1}{1+t^{2}} \geq \frac{1}{2+t^{2}} \geq 0$ for all $t \geq 0$, we have

$$
\frac{1}{2} \leq r(t) \leq q(t)
$$

for all $t \geq 0$, where $\delta_{1}=\frac{1}{2}>0$.

(ii) Differentiating the functions $q(t)$ and $r(t)$ with respect to $t$, we obtain

$$
\dot{q}(t)=\frac{-2 t}{\left(1+t^{2}\right)^{2}} \text { and } \dot{r}(t)=\frac{-2 t}{\left(2+t^{2}\right)^{2}} .
$$

Now, since $\frac{-2 t}{\left(1+t^{2}\right)^{2}} \leq \frac{-2 t}{\left(2+t^{2}\right)^{2}} \leq 0$ for all $t \geq 0$, we obtain

$$
\dot{q}(t) \leq \dot{r}(t) \leq 0
$$

for all $t \geq 0$.

(e) It is not difficult to show that the function $p(t, x, y, z)$ satisfies the integral inequality

$$
\int_{0}^{\infty}\left|\frac{1}{1+t^{2}+x^{2}+y^{2}+z^{2}}\right| d t<\infty
$$

for all $t \geq 0, x, y$ and $z$. Hence, all the assumptions of the theorems are satisfied and the conclusions follow.

Acknowledgment. The authors would like to thank the referee for his or her valuable advice, suggestions and encouragement.

\section{References}

[1] Ademola, A. T. and Arawomo, P. O.; Boundedness and stability of solutions of some nonlinear differential equations of the third order, The Pacific Journal of Science and Technology., 10, (2), pp. 187-193, (2009).

[2] Ademola, A. T. and Arawomo, P. O.; On the stability and ultimate boundedness of solutions for certain third order differential equations. Journal of Mathematics and Statistic., 4, pp. 202-208, (2008). 
[3] Ademola, A. T. and Arawomo, P. O.; Stability and ultimate boundedness of solutions to certain third order differential equations. Applied Mathematics E-Notes., 10, pp. 61-69, (2010).

[4] Ademola, A. T. and Arawomo, P. O.; Stability and uniform ultimate boundedness of solutions of some third order differential equations. Acta Mathematica Academiae Paedagogicae Nyíregyháziensis, 27, pp. 51-59, (2011).

[5] Ademola, A. T., Ogundiran, M.O., Arawomo, P.O. and Adesina, O.A.; Boundedness results for a certain third order nonlinear differential equations. Appl. Math. Comput. 216, pp. 3044-3049, (2010).

[6] Ademola, A. T., Ogundiran, M.O., Arawomo, P.O. and Adesina, O.A.; Stability results for the solutions of a certain third order nonlinear differential equation. Mathematical Sciences Research Journal MSRJ. Vol. 12, no. 6, pp. 124-134, (2008).

[7] Afuwape, A. U. and Adesina, O.A.; On the bounds for mean-values of solutions to certain third order nonlinear differential equations, Fasciculi Mathematici, Vol. 36, pp. 5-14, (2005).

[8] Afuwape, A. U. and Omeike, M.O.; Convergence of solutions for certain non-homogeneous third order differential equations. Kragujevac J. Math., 31, pp. 5-16, (2008).

[9] Chukwu, E. N.; On boundedness of solutions of third order differential equations, Ann. Mat. Pura. Appl., 104, (4), pp. 123-149, (1975).

[10] Ezeilo, J. O. C.; A note on a boundedness theorem for some third order differential equations, J. London Math. Soc., 36, pp. 439-444, (1961).

[11] Ezeilo, J. O. C.; A boundedness theorem for a certain third order differential equation, Proc. London Math. Soc. (3), 13, pp. 99-124, (1963).

[12] Ezeilo, J. O. C.; A generalization of a boundedness theorem for the equation $x+\alpha \ddot{x}+\phi_{2}(\dot{x})+\phi_{3}(x)=\psi(t, x, \dot{x}, \ddot{x})$, Atti. Accad. Naz. Lincei Rend. Cl. Sci. Fis. Mat. Natur., (13), 50, pp. 424-431, (1971).

[13] Ezeilo, J. O. C.; A generalization of some boundedness results by Reissig and Tejumola, J. Math. Anal. Appl., 41, pp. 411-419, (1973). 
[14] Ezeilo, J. O. C. and Tejumola, H.O.; Boundedness theorems for certain third order differential equations, Atti. Accad. Naz. Lincei Rend. Cl. Sci. Fis. Mat. Natur., (10), 55, pp. 194-201, (1973).

[15] Ezeilo, J. O. C.; Further results for the solutions of a third order differential equation, Proc. Camb. Phil. Soc., 59, pp. 111-116, (1963).

[16] Lakshmikantham, V. and Leels, S.; Ordinary differential and integral inequalities: Theory and applications Vol. 1 Academic Press New York, (1969).

[17] Nakashima, M.; Asymptotic behaviour of solutions of some third order differential equations. Rep. Fac. Sci. Kagoshima Uni. (Math. Phys. Chem.) No.4, pp. 7-15, (1971).

[18] Ogundare, B. S.; On the convergence of solutions of certain third order nonlinear differential equation, Math.Sci. Res. J. 9 (11), pp. 304-312, (2005).

[19] Omeike, M. O.; New results on the asymptotic behaviour of a third order nonlinear differential equations, Differential Equations \& Applications, pp. 1-13, (2008).

[20] Qian, C.; Asymptotic behaviour of a third order nonlinear differential equation, J. Math. Anal. Appl., 284, no.1, pp. 191-205, (2003).

[21] Reissig, R., Sansone, G. and Conti, R.; Nonlinear differential equations of higher order, Noordhoff International Publishing Leyeden, (1974).

[22] Rouche, N., Habets, N. and Laloy, M.; Stability theory by Liapunov's direct method. Applied Mathematical Sciences 22, Springer-Verlag New York. Heidelberg. Berlin, (1977).

[23] Swick, K. E.; Asymptotic behaviour of the solutions of certain third order differential equations, SIAM J. Appl.,, 19, N0. 1, pp. 96-102, (1970).

[24] Swick, K. E.; On the boundedness and the stability of solutions for some non-autonomous differential equations of the third order, J. London Math. Soc., 44, pp. 347-359, (1969).

[25] Tejumola, H. O.; A note on the boundedness of solutions of some nonlinear differential equations of the third order, Ghana J. of Science, 11 (2), pp. 117-118, (1970). 
[26] Tunç, C.; Boundedness of solutions of a third order nonlinear differential equation, J. Inequal. Pure and Appl. Math., 6 (1), pp. 1-6, (2005).

[27] Tunç, C.; On the asymptotic behaviour of solutions of certain third order nonlinear differential equations, J. Applied Math. and Stochastic Anal., 1, pp. 29-35, (2005).

[28] Tunç, E.; On the convergence of solutions of certain third order differential equations. Discrete Dyn. Nature and Society., pp. 1-12, (2009).

[29] Yoshizawa, T.; Stability theory by Liapunov's second method, The Mathematical Society of Japan (1966).

\author{
A. T. Ademola \\ Department of Mathematics, \\ University of Ibadan, \\ Ibadan, \\ Nigeria \\ e-mail : ademola672000@yahoo.com \\ and
}

\title{
P.O. Arawomo
}

Department of Mathematics, University of Ibadan, Ibadan, Nigeria

e-mail : womopeter@gmail.com 